\title{
Resistive-wall wake for nonrelativistic beams revisited
}

\author{
Gennady Stupakov® \\ SLAC National Accelerator Laboratory, Menlo Park, California 94025, USA
}

(Received 26 June 2020; accepted 21 August 2020; published 8 September 2020)

\begin{abstract}
In this paper, we derive the longitudinal and transverse resistive wall impedance for a beam traveling in a round pipe with $v<c$. We argue that from the general formulas for the impedance obtained from the solution of the Maxwell equations one has to subtract the space charge component. After such subtraction, we find that the classical expressions for the impedance derived in the ultrarelativistic limit are also applicable for long bunches even when they are nonrelativistic - in contrast to the conclusion of Zimmermann and Oide [Phys. Rev. Accel. Beams 7, 044201 (2004)]. We also calculate the resistive wall Green-function wake for $v<c$ at small distances and show that the jump at the origin in the longitudinal wake and in the derivative of the transverse wake are smeared out and the wake propagates in front of the source charge.
\end{abstract}

DOI: 10.1103/PhysRevAccelBeams.23.094401

\section{INTRODUCTION}

Resistive wall (RW) wakefield plays an important, often dominant, role in modern accelerators, especially in those with a small transverse size of the vacuum chamber. Calculations of this wake go back to the 1960s [1] and, in the relativistic limit, $\gamma \rightarrow \infty$ ( $\gamma$ is the Lorentz factor), the results are well established and covered in textbooks [2,3]. For nonrelativistic particles, the derivation has been revised relatively recently in a number or publications, with the most thorough work reported in Refs. [4,5]. The authors of Ref. [5] (to which we will refer as $\mathrm{ZO}$ in what follows) note however that their results do not fully agree with an earlier paper [4].

Upon thorough examination of Ref. [5], we found that $\mathrm{ZO}$ results are not fully satisfactory. The authors made an expansion of the impedance and derived corrections to the infinite- $\gamma$ Green-function wake. However, these corrections scale as $|z|^{-7 / 2}$ for the longitudinal and $|z|^{-5 / 2}$ for the transverse wake, where $z$ is the distance between the source and the test particles. In applications, this wake should be used in a convolution integral with the bunch distribution, but it leads to expressions that diverge as $z \rightarrow 0$, so these corrections do not make sense. ${ }^{1}$ When, in the last section of

Published by the American Physical Society under the terms of the Creative Commons Attribution 4.0 International license. Further distribution of this work must maintain attribution to the author(s) and the published article's title, journal citation, and DOI.

${ }^{1}$ The longitudinal Green-function wake in the limit $\gamma=\infty$ scales as $|z|^{-3 / 2}$ and also formally diverges at $z \rightarrow 0$. There exists however a proof that shows how to remove this divergence by integration by parts. No such proof is given by $\mathrm{ZO}$ as to how to treat nonintegrable wakes in their paper. the paper, the authors apply these results to four practical cases, they conclude that "the deviations from the classical ultrarelativistic expression can amount to 1 or 2 orders of magnitude." In the present paper, we arrive at the opposite conclusion-in all four cases the results of the nonrelativistic wake is very close to the relativistic one.

While our calculations follow close those of $\mathrm{ZO}$, the crucial element where we diverge is the understanding that a formally calculated impedance contains not only a contribution from the resistive wall, but also from the space charge. We define the space charge part as the impedance in the same pipe in the limit of perfect conductivity. We then subtract this impedance from the full expression to obtain the resistive wall part. A similar approach has been advocated in the literature [6] for somewhat different problem of the impedance of a short resistive wall insert. Naturally, the so defined RW impedance vanishes in the limit of perfect conductivity.

We use the Gaussian system of units throughout this paper. To convert our expressions for the impedance and wake to the MKS system they should be multiplied by the factor $Z_{0} c / 4 \pi$; in the case of the conductivity, the cgs value is divided by the factor $Z_{0} c / 4 \pi$.

\section{LONGITUDINAL IMPEDANCE}

Following $\mathrm{ZO}$, we consider a beam pipe of radius $b$ with the beam charge line density $\lambda_{b}$, traveling at the center of the beam pipe with frequency $\omega=k v$ and velocity $v<c$,

$$
\lambda_{b} e^{-i \omega t+i k z}
$$

where $z$ is the longitudinal coordinate along the axis of the pipe. Solving Maxwell's equations in the cylindrical coordinate system $r, \theta, z$, we find the magnetic and the 
longitudinal electric fields in the pipe (all the fields depend on $z$ and $t$ as $e^{-i \omega t+i k z}$, so we drop this factor from the expressions below),

$$
\begin{aligned}
& B_{\theta}=-c p k_{r} I_{1}\left(k_{r} r\right)+c q k_{r} K_{1}\left(k_{r} r\right), \\
& E_{z}=-\frac{i \omega}{\beta^{2} \gamma^{2}}\left[p I_{0}\left(k_{r} r\right)+q K_{0}\left(k_{r} r\right)\right],
\end{aligned}
$$

where $q=2 \lambda_{b} v / c^{2}$ and $k_{r}=\omega / c \beta \gamma$. The parameter $p$ is this equation will be found from the boundary condition on the wall.

In contrast to ZO, where the authors solve the Maxwell equations inside the metal wall of the pipe and then take the limit of the skin depth much smaller than the pipe radius, we arrive at the same result using the Leontovich boundary condition on the wall [7],

$$
E_{z}=-\zeta B_{\theta}
$$

with

$$
\zeta=(1-i) \sqrt{\frac{\omega}{8 \pi \sigma}}=\frac{1}{2}(1-i) \frac{\omega}{c} \delta_{\text {skin }},
$$

where $\sigma$ is the metal conductivity (here, and in what follows, we assume positive frequencies, $\omega>0$ ), and the skin depth $\delta_{\text {skin }}=c(2 \pi \sigma \omega)^{-1 / 2}$. We emphasize here that using the Leontovich boundary condition we limit our analysis by the requirement $\delta_{\text {skin }} \ll b$, which is well satisfied in many practical applications. Substituting Eqs. (2) into (3) and solving it for $p$ gives

$$
p=q \frac{c \zeta k_{r} K_{1}\left(k_{r} b\right)-i \omega K_{0}\left(k_{r} b\right) / \beta^{2} \gamma^{2}}{c \zeta k_{r} I_{1}\left(k_{r} b\right)+i \omega I_{0}\left(k_{r} b\right) / \beta^{2} \gamma^{2}} .
$$

To calculate the longitudinal impedance, $Z_{\|}=-E_{z} / \lambda_{b} v$, we need to take the longitudinal field on the axis, $r=0$. In doing so, we discard the second term in the expression (2) for $E_{z}$, which is the electric field of a line charge in free space and is singular at $r=0$. We then obtain

$$
\begin{aligned}
Z_{\|} & =i \omega \frac{2}{c^{2} \beta^{2} \gamma^{2}} \frac{c \zeta k_{r} K_{1}\left(k_{r} b\right)-i \omega K_{0}\left(k_{r} b\right) / \beta^{2} \gamma^{2}}{c \zeta k_{r} I_{1}\left(k_{r} b\right)+i \omega I_{0}\left(k_{r} b\right) / \beta^{2} \gamma^{2}} \\
& =i \omega \frac{2}{c^{2} \beta^{2} \gamma^{2}} \frac{\omega^{2} \delta_{\text {skin }} K_{1}\left(k_{r} b\right)+(1-i) c^{2} k_{r} K_{0}\left(k_{r} b\right)}{\omega^{2} \delta_{\text {skin }} I_{1}\left(k_{r} b\right)-(1-i) c^{2} k_{r} I_{0}\left(k_{r} b\right)} .
\end{aligned}
$$

This expression agrees with Eq. (54) in Ref. [5] if we correct their equation inserting $k_{r}$ in front of $K_{0}$ and $I_{0}$ functions (without this correction Eq. (54) has wrong dimensions of the summands in the numerator and the denominator).
Equation (6) is not yet our final result: as explained in the Introduction, it is a combination of the resistive wall impedance and the space charge impedance in a pipe of radius $b$. We need to subtract the latter from Eq. (6). It makes sense to define the space charge impedance as the limit of perfect conductivity, that is when $\sigma \rightarrow \infty$ or, equivalently, $\zeta \rightarrow 0$. Taking this limit in Eq. (6) we obtain the space charge impedance,

$$
Z_{\|, s c}=\lim _{\zeta \rightarrow 0} Z_{\|}=-i \omega \frac{2}{c^{2} \beta^{2} \gamma^{2}} \frac{K_{0}\left(k_{r} b\right)}{I_{0}\left(k_{r} b\right)} .
$$

Subtracting Eq. (7) from Eq. (6) gives

$Z_{\|, \mathrm{rw}}=Z_{\|}-Z_{\|, s c}=\frac{2 i \zeta}{c b} \frac{1}{\left[\beta \gamma \zeta I_{1}\left(k_{r} b\right)+i I_{0}\left(k_{r} b\right)\right] I_{0}\left(k_{r} b\right)}$.

To arrive at Eq. (8) we used the mathematical identity

$$
I_{0}(x) K_{1}(x)+K_{0}(x) I_{1}(x)=\frac{1}{x} .
$$

In application to short bunches typical for modern electron linear accelerators it is convenient to introduce the parameter $s_{0}$ (see Ref. [8]),

$$
s_{0}=\left(\frac{c b^{2}}{2 \pi \sigma}\right)^{1 / 3}
$$

normalize the frequency by the ratio $c / s_{0}$,

$$
\varkappa=\frac{\omega s_{0}}{c},
$$

and use the dimensionless parameter

$$
\nu=\frac{b}{s_{0} \beta \gamma} .
$$

The parameter $s_{0}$ is usually small: for an aluminum pipe $\left(\sigma=3.28 \times 10^{17} \mathrm{~s}^{-1}\right.$ which is equivalent to $3.65 \times$ $10^{7} \Omega^{-1} \mathrm{~m}^{-1}$ in the MKS system) of radius $b=5 \mathrm{~cm}$ it is equal to $71 \mu \mathrm{m}$. In terms of the new variables $\varkappa$ and $\nu$, Eq. (8) reads,

$$
Z_{\|, \mathrm{rw}}=\frac{2 s_{0}}{c b^{2}} \frac{i \varkappa^{1 / 2} \nu}{I_{0}(\varkappa \nu)\left[\sqrt{\varkappa} I_{1}(\varkappa \nu)+(i-1) \nu I_{0}(\varkappa \nu)\right]} .
$$

We can recover the known impedance [2] in the limit $\gamma \rightarrow \infty$ assuming $k_{r} b \ll 1$ and using the approximations

$$
I_{1}\left(k_{r} b\right) \approx \frac{1}{2} k_{r} b, \quad I_{0}\left(k_{r} b\right) \approx 1,
$$

which gives 


$$
Z_{\|, \mathrm{rw}}=\frac{2}{c b} \frac{1}{1 / \zeta-i \omega b / 2 c}=\frac{2 s_{0}}{c b^{2}} \frac{1}{(1+i) \varkappa^{-1 / 2}-i \varkappa / 2} .
$$

The second term in the denominator of Eq. (15) can be neglected in comparison with the first one in the limit of small frequencies, $x \ll 1$, and in this limit we arrive at the classical expression for the impedance valid for bunches with the bunch length $\sigma_{z} \gg s_{0}$,

$$
Z_{\|, \mathrm{rw}}=(1-i) \frac{s_{0}}{c b^{2}} \varkappa^{1 / 2} .
$$

It is important to realize here that the product $k_{r} b=$ $\omega b / c \beta \gamma$ becomes small not only in the limit of large values of $\gamma$, but also for $\gamma \sim 1$ if the frequency is small, $\omega / c \ll \beta / b$. This means, somewhat surprisingly, that relativistic formulas for the resistive wall wake can also be used for nonrelativistic beams if the bunch lengths is large enough, $\sigma_{z} \gg b / \gamma$. We will see in Sec. IV that this is the case for the ZO numerical examples. Another observation that follows from the analysis of the smallness of $k_{r} b$ is that the resistive wall wake derived in the limit $\gamma=\infty$ may not be applicable even for $\gamma \gg 1$ if the bunch is very short, $\sigma_{z} \lesssim b / \gamma$. This requirement is easy to understand if we notice that the Coulomb field of a relativistic point charge moving in a pipe of radius $b$ projects its electric and magnetic fields on the wall within an angle $\sim 1 / \gamma$ with the spot size of the order of $b / \gamma$. We will explore this observation in more detail in Sec. IV.

\section{TRANSVERSE IMPEDANCE}

To calculate the transverse impedance, we consider two line-charges as given by Eq. (1) but with opposite signs, $+\lambda_{b}$ and $-\lambda_{b}$, shifted off axis in the horizontal plane by the distance $\pm \Delta / 2$, respectively, where $\Delta \ll b$ (more precisely, mathematically we solve the problem with $\Delta \rightarrow 0, \lambda_{b} \rightarrow \infty$, but their product $\lambda_{b} \Delta$ remaining constant). The dipole momentum per unit length of these two line charges is

$$
\xi_{b} e^{-i \omega t+i k z},
$$

where $\xi_{b}=\lambda_{b} \Delta$. In the cylindrical coordinate system $r, \theta$, $z$, the dependence of the components of the electromagnetic field versus $t, z$ and $\theta$ is given by the factor $e^{-i \omega t+i k z+i \theta}$ (which will omit in what follows), and the radial dependence of the fields inside the pipe is determined from the Maxwell equations. The solution contains four arbitrary constants. Two of these constants are found from the requirement that the field in the limit $r \rightarrow 0$ approaches the Coulomb field of a line-dipole, $\lim _{r \rightarrow 0} E_{r}=2 \xi_{b} / r^{2}$ and $\lim _{r \rightarrow 0} B_{z}=0$. The other two constants are found from Eq. (3) supplemented by another component of the vectorial Leontovich boundary condition, $E_{\theta}=\zeta B_{z}$.
From the total electromagnetic field one has to subtract the field of the line-dipole in free space (which can be formally found taking the limit $b \rightarrow \infty$ ) and to use the difference to calculate the transverse impedance,

$$
Z_{\perp}=-\left.\frac{i}{\xi_{b} v}\left[E_{r}(r)-\frac{v}{c} B_{\theta}(r)\right]\right|_{r=0} .
$$

As in the longitudinal case, this impedance consists of the combination of the space charge and the resistive wall transverse impedance and we need to separate them as we did in the previous section by associating the space charge impedance $Z_{\perp, s c}$ with the value of $Z_{\perp}$ at $\zeta=0$, and defining $Z_{\perp, r w}=Z_{\perp}-Z_{\perp, s c}$. The result is a rather complicated expression,

$$
Z_{\perp, r w}=\frac{N}{D}
$$

where

$$
\begin{aligned}
N= & \zeta k^{2}\left[I_{1}\left(k_{r} b\right)\left(b \zeta k+i \gamma^{2} \beta\right)-i b \gamma k \beta I_{0}\left(k_{r} b\right)\right], \\
D= & c \gamma^{2} I_{1}\left(k_{r} b\right)\left\{I_{1}\left(k_{r} b\right)^{2}\left[b^{2} \zeta k^{2}+i b \gamma^{2}\left(\zeta^{2}+1\right) k \beta+\gamma^{2} \zeta\right]\right. \\
& -b^{2} \gamma^{2} \zeta k^{2} \beta^{2} I_{0}\left(k_{r} b\right)^{2} \\
& \left.-i b \gamma k \beta I_{1}\left(k_{r} b\right) I_{0}\left(k_{r} b\right)\left[b\left(\zeta^{2}+1\right) k+2 i \gamma^{2} \zeta \beta\right]\right\}, \quad(20)
\end{aligned}
$$

with $k=\omega / v$. This expression can be slightly simplified if one takes into account that the parameter $\zeta$, being a ratio of the skin depth to the wavelength $c / \omega$, is supposed to be much smaller than one, hence $1+\zeta^{2} \approx 1$. Using this approximation, we rewrite Eqs. (20), now expressing them through the variables $\varkappa, \nu$, and $s_{0}$,

$$
\begin{aligned}
N= & 2 i \gamma^{2} \varkappa^{3} \nu \beta^{2} I_{0}(\varkappa \nu) \\
& -(1-i) \varkappa^{2} I_{1}(\varkappa \nu)\left[\varkappa^{3 / 2}-(1-i) \gamma^{2} \beta^{2}\right], \\
D= & 2 c \gamma^{4} s_{0}^{2} \beta^{3} I_{1}(\varkappa \nu)\left\{\gamma^{2} \varkappa^{2} \nu^{2} \beta^{2} I_{0}(\varkappa \nu)^{2}\right. \\
& +I_{1}(\varkappa \nu)^{2}\left[-\varkappa^{2} \nu^{2}+(1-i) \gamma^{2} \sqrt{\varkappa} \nu^{2} \beta^{2}-1\right] \\
& \left.-(1-i) \gamma^{2} \varkappa \nu \beta^{2}\left[\sqrt{\varkappa} \nu^{2}+(1+i)\right] I_{0}(\varkappa \nu) I_{1}(\varkappa \nu)\right\} .
\end{aligned}
$$

Taking the limit $\gamma \rightarrow \infty$ one obtains

$$
\begin{aligned}
Z_{\perp, r w} & =\frac{8 i \zeta}{c b^{2}\left(b^{2} \zeta \varkappa^{2}+2 i b \varkappa-2 \zeta\right)} \\
& =\frac{4}{c b^{2}}\left(b k(1+i) \sqrt{2 \pi \frac{\sigma}{\omega}}-\frac{1}{2} i b^{2} k^{2}+i\right)^{-1},
\end{aligned}
$$

which is the classical result that can be found in the textbook [2] (here $k=\omega / c$ ). The result (22) can be even more simplified in the limit $k \ll 1 / s_{0}$,

$$
Z_{\perp, r w}=\frac{8 i \zeta}{c b^{2}(2 i b \varkappa)}=\frac{4}{c b^{2}}\left(b k(1+i) \sqrt{2 \pi \frac{\sigma}{\omega}}\right)^{-1} .
$$


TABLE I. Paramters of four accelerators from Ref. [5].

\begin{tabular}{lcccc}
\hline \hline & SNS & J-PARC & PS booster & ECR \\
\hline$\gamma$ & 2.1 & 1.4 & 1.05 & 1.003 \\
$\sigma_{z}$ & $25 \mathrm{~m}$ & $12 \mathrm{~m}$ & $26 \mathrm{~m}$ & $100 \mathrm{~m}$ \\
$b$ & $8 \mathrm{~cm}$ & $12.5 \mathrm{~cm}$ & $30 \mathrm{~cm}$ & $3 \mathrm{~cm}$ \\
$\delta_{\text {skin }} / b$ & $4.1 \times 10^{-3}$ & $2.0 \times 10^{-3}$ & $1.8 \times 10^{-3}$ & $7.4 \times 10^{-2}$ \\
$b / \sigma_{z} \gamma$ & $1.5 \times 10^{-3}$ & $7.4 \times 10^{-3}$ & $1.0 \times 10^{-2}$ & $2.9 \times 10^{-4}$ \\
\hline \hline
\end{tabular}

\section{APPLICATIONS}

We will now apply our results to the four accelerators considered by ZO. They are: the accumulator ring of the Spallation Neutron Source (SNS), the 3-GeV rapidcycling synchrotron of the Japan Proton Accelerator Research Complex (J-PARC), the booster rings of the CERN Proton Synchrotron (PS booster), and an electroncyclotron resonance (ECR) ion source. The parameters relevant for our calculations are taken from Table IV of Ref. [5] and listed in Table I. Following ZO we assumed a stainless steel conductivity of the pipe with $\sigma=1.25 \times$ $10^{16} \mathrm{~s}^{-1}$ ( $\sigma=1.4 \times 10^{6} \Omega^{-1} \mathrm{~m}^{-1}$ in the MKS system). In line four of the table we indicate the ratio of the skin depth at the characteristic frequency of the bunch $\omega \sim v / \sigma_{z}$ to the pipe radius - the small value of this parameters justifies the usage of the impedance derived with the Leontovich boundary condition. The last line shows the parameter $b / \sigma_{z} \gamma$ which is an estimate of the argument $k_{r} b$ in Eq. (8) when the frequency $\omega$ is evaluated as $\omega \sim v / \sigma_{z}$. As was discussed in Sec. II, if this parameter is small, one can use the classical formulas for the resistive wall wake derived in the limit $\gamma=\infty$. We see that for all four examples this parameter is extremely small, even though the parameter $\gamma$ is not large-the smallness is provided by the large bunch length relative to the radius of the pipe. Plotting the bunch wakefield for all four cases, we found, as expected, that the wake calculated with a nonrelativistic formula (8) is the same as the one calculated using Eq. (16). One of such plots is shown in Fig. 1(a), where the SNS bunch wake is calculated assuming a Gaussian profile and using the formula

$$
W_{\|, \mathrm{rw}}(z)=\frac{1}{2 \pi} \int_{-\infty}^{\infty} d \omega Z_{\|, \mathrm{rw}}(\omega) e^{-i \omega z / v} e^{-\omega^{2} \sigma_{z}^{2} / 2 v^{2}}
$$

We also calculated the SNS transverse wake,

$W_{\perp, \mathrm{rw}}(z)=\frac{i}{2 \pi} \int_{-\infty}^{\infty} d \omega Z_{\perp, \mathrm{rw}}(\omega) e^{-i \omega z / v} e^{-\omega^{2} \sigma_{z}^{2} / 2 v^{2}}$

with the exact expression for the transverse impedance (21) and its ultrarelativistic approximation (23). These two wakes are plotted in Fig. 1(b), and, as one can see, they also go on top of each other.

An interesting example where the nonrelativistic approximation indeed deviates from its limit $\gamma=\infty$ is presented by the Green-function wake of a relativistic point charge at a small distance from the source charge. As is well known, in the limit $\gamma=\infty$, the longitudinal wake is zero in front of the charge, $z<0$, and it jumps to the value $4 / b^{2}$ immediately behind it, $z=0^{+}$. The transverse wake is continuous at $z=0$, but has a jump of its derivative, $d w_{\perp} / d z$, from zero at $z=0^{-}$to $8 / b^{4}$ at $z=0^{+}$. In the Fourier representation, theses jumps are formed by high frequency components of the spectrum, and hence by large values of the argument $k_{r} b$ of the Bessel functions in Eqs. (8) and (20). As was discussed in Sec. II, these frequencies should be described by the equations which do not assume $\gamma=\infty$. To make a quantitative illustration of this point in Fig. 2 we show the plots of the longitudinal point charge wakefield for three different values of the parameter $\nu, \nu=0.1,0.5,1.0$, together with the curve corresponding to $\nu=0$ (that is $\gamma=\infty$ ). The latter is discontinuous at $z=0$. In Fig. 3 a similar plot is shown for the transverse resistive wall wake. One can see how the wake deviates from its limiting profile $\gamma=\infty(\nu=0)$ with

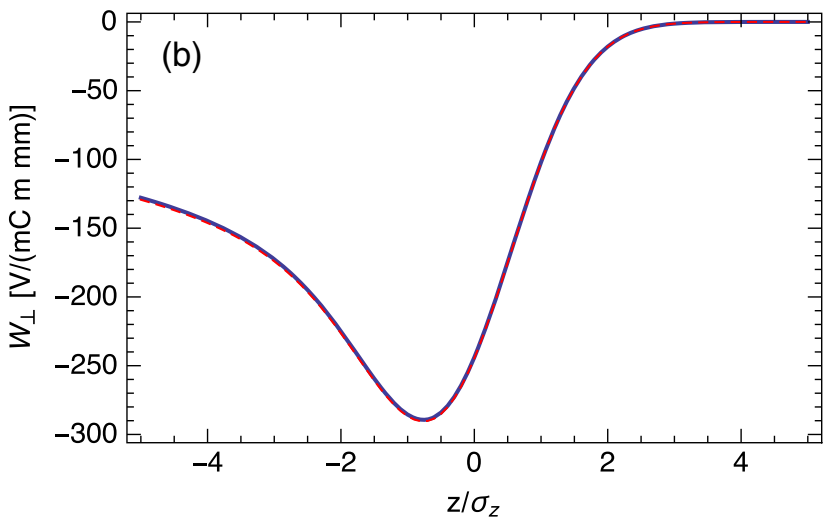

FIG. 1. SNS longitudinal (a) and transverse (b) RW bunch wakes. On each plot there are two lines that go on top of each other: one line is calculated with a nonrelativistic formulas (8) and (21) and the other one with Eqs. (16) and (23), respectively. 


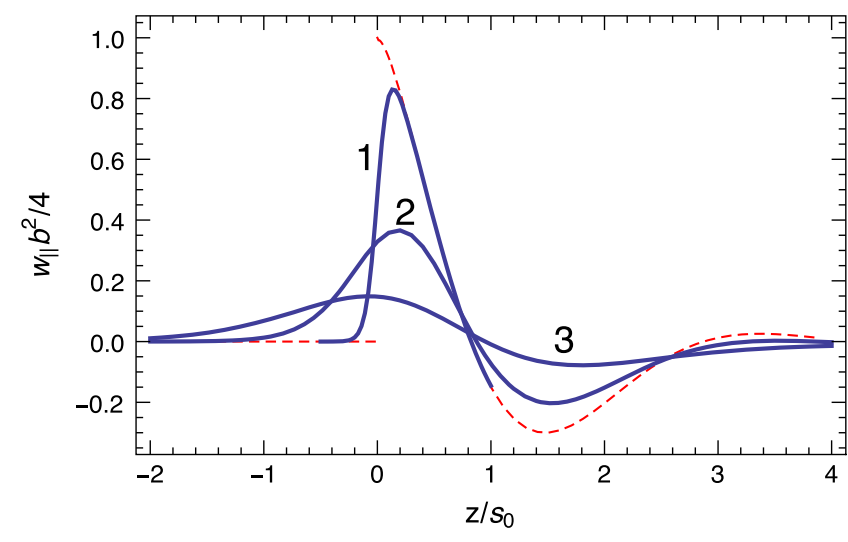

FIG. 2. The longitudinal wake of a point charge for three different values of the parameter $\nu$ : (1) $\nu=0.1$, (2) $\nu=0.5$, and (3) $\nu=1$. The red dashed line shows the limit $\nu=0$ when the wake is equal to zero in front of the charge $(z<0)$ and then jumps to the value $4 / b^{2}$ at $z=0^{+}$.

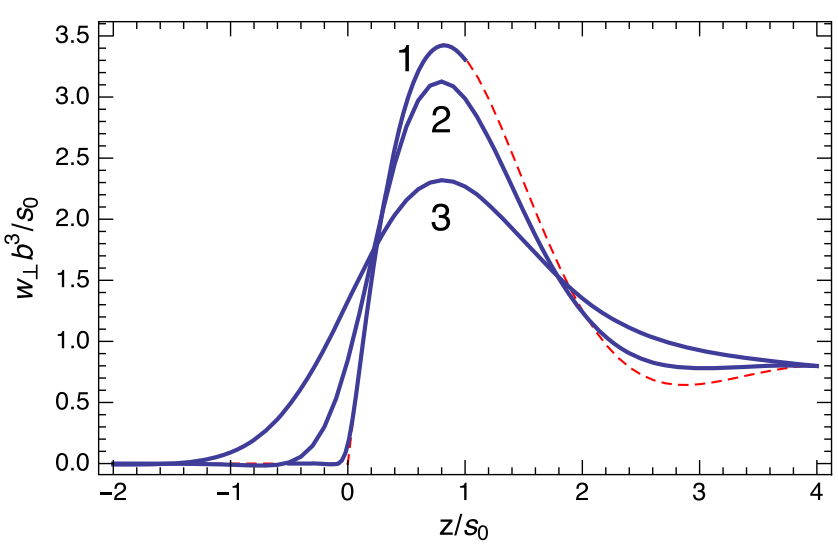

FIG. 3. The transverse wake of a point charge for three different values of the parameter $\nu$ : (1) $\nu=0.1,(2) \nu=0.5$, and (3) $\nu=1$. The red dashed line shows the limit $\nu=0$.

increasing value of $\nu$. Note also how these wakes propagate ahead of the source charge $(z<0)$, which of course is expected if one drops the assumption that the charge that creates the wake moves with $v=c$.

\section{SUMMARY}

In this paper we revised the previously derived expressions for the longitudinal and transverse RW impedance in a round pipe. We advocated that solving Maxwell's equations gives an impedance that is a sum of the resistive wall and the space charge components. Subtracting the space charge, we arrived at the expressions which in the limit of $\gamma \rightarrow \infty$ reduce to the classical RW formulas. We also observed that the parameter that controls the transition to the limit $\gamma=\infty$ is $\omega b / c \beta \gamma$. This parameter becomes small not only in the limit of large values of $\gamma$, but also for small values of $\omega$. Specifically, for long bunches with $\sigma_{z} \gg b / \gamma$ one can use the RW formulas derived for infinitely large $\gamma$, even when $\gamma \sim 1$. We have shown, by direct numerical integration, that for four examples from Ref. [5], the RW wakefield calculated with exact formulas is indistinguishable from the one calculated with the ultrarelativistic approximation, in contrast to the conclusion of ZO. Finally, we demonstrated how a point charge wake at short distances starts to deviate from its limiting ultrarelativistic profile when the parameter $\nu$ increases from zero to a finite value of the order of one.

\section{ACKNOWLEDGMENTS}

The author would like to thank K. Bane and K. Oide for useful discussions. This work was supported by the Department of Energy, Contract No. DE-AC0376 SF00515.

[1] P. L. Morton, V. K. Neil, and A. M. Sessler, Wake fields of a pulse of charge moving in a highly conducting pipe of circular cross section, J. Appl. Phys. 37, 3875 (1966).

[2] A. W. Chao, Physics of Collective Beam Instabilities in High Energy Accelerators (Wiley, New York, 1993).

[3] A. Wolski, Beam Dynamics in High Energy Particle Accelerators (Imperial College Press, London, UK, 2014).

[4] A. M. Al-khateeb, O. Boine-Frankenheim, I. Hofmann, and G. Rumolo, Analytical calculation of the longitudinal space charge and resistive wall impedances in a smooth cylindrical pipe, Phys. Rev. E 63, 026503 (2001).

[5] F. Zimmermann and K. Oide, Resistive-wall wake and impedance for nonultrarelativistic beams, Phys. Rev. Accel. Beams 7, 044201 (2004).

[6] Y. Shobuda, Y. H. Chin, and K. Takata, Coupling impedances of a resistive insert in a vacuum chamber, Phys. Rev. Accel. Beams 12, 094401 (2009).

[7] L. D. Landau and E. M. Lifshitz, Electrodynamics of Continuous Media, 2nd ed., Course of Theoretical Physics, Vol. 8 (Pergamon, London, 1984) (Translated from Russian).

[8] K. L. F. Bane and M. Sands, The short-range resistive wall wakefields, in Micro Bunches Workshop, AIP Conference Proceedings No. 367, edited by E. B. Blum, M. Dienes, and J. B. Murphy (AIP, New York, 1996), p. 131. 